\title{
Chronic exposure to haloperidol and olanzapine leads to common and divergent shape changes in the rat hippocampus in the absence of grey-matter volume loss
}

\author{
W. R. Crum ${ }^{1}$ t, F. Danckaers ${ }^{2}$ t, T. Huysmans ${ }^{2}$, M.-C. Cotel $^{3}$, S. Natesan ${ }^{3}$, M. M. Modo ${ }^{4}$, J. Sijbers ${ }^{2}$, \\ S. C. R. Williams ${ }^{1}$, S. Kapur ${ }^{3}$ and A. C. Vernon ${ }^{3,4 *}$ \\ ${ }^{1}$ Department of Neuroimaging, King's College London, Institute of Psychiatry, Psychology and Neuroscience, Centre for Neuroimaging Sciences, De \\ Crespigny Park, London, UK \\ ${ }^{2}$ Department of Physics, iMinds-Vision Laboratory, University of Antwerp, Antwerp, Belgium \\ ${ }^{3}$ Department of Psychosis Studies, King's College London, Institute of Psychiatry, Psychology and Neuroscience, De Crespigny Park, London, UK \\ ${ }^{4}$ Department of Basic and Clinical Neuroscience, King's College London, Institute of Psychiatry, Psychology and Neuroscience, Maurice Wohl \\ Institute for Clinical Neuroscience, London, UK
}

Background. One of the most consistently reported brain abnormalities in schizophrenia (SCZ) is decreased volume and shape deformation of the hippocampus. However, the potential contribution of chronic antipsychotic medication exposure to these phenomena remains unclear.

\begin{abstract}
Method. We examined the effect of chronic exposure (8 weeks) to clinically relevant doses of either haloperidol (HAL) or olanzapine (OLZ) on adult rat hippocampal volume and shape using ex vivo structural MRI with the brain retained inside the cranium to prevent distortions due to dissection, followed by tensor-based morphometry (TBM) and elastic surfacebased shape deformation analysis. The volume of the hippocampus was also measured post-mortem from brain tissue sections in each group.

Results. Chronic exposure to either HAL or OLZ had no effect on the volume of the hippocampus, even at exploratory thresholds, which was confirmed post-mortem. In contrast, shape deformation analysis revealed that chronic HAL and OLZ exposure lead to both common and divergent shape deformations ( $q=0.05$, FDR-corrected $)$ in the rat hippocampus. In particular, in the dorsal hippocampus, HAL exposure led to inward shape deformation, whereas OLZ exposure led to outward shape deformation. Interestingly, outward shape deformations that were common to both drugs occurred in the ventral hippocampus. These effects remained significant after controlling for hippocampal volume suggesting true shape changes.
\end{abstract}

Conclusions. Chronic exposure to either HAL or OLZ leads to both common and divergent effects on rat hippocampal shape in the absence of volume change. The implications of these findings for the clinic are discussed.

Received 22 October 2015; Revised 23 June 2016; Accepted 23 June 2016; First published online 12 August 2016

Key words: antipsychotic, hippocampus, magnetic resonance imaging, schizophrenia, shape, volume.

\section{Introduction}

Large-scale magnetic resonance imaging (MRI) studies of patients with schizophrenia (SCZ) commonly report decreases in hippocampal volume with robust effect sizes (van Erp et al. 2015). Advances in computational neuroanatomical techniques have refined this to include descriptions of progressive shape deformations

\footnotetext{
* Address for correspondence: Dr A. C. Vernon, Department of Basic and Clinical Neuroscience, King's College London, Institute of Psychiatry, Psychology and Neuroscience, Maurice Wohl Institute for Clinical Neuroscience, 5 Cutcombe Road, London SE5 9RT, UK.

(Email: anthony.vernon@kcl.ac.uk)

+ These authors contributed equally to this work.
}

that also occur in the hippocampus of SCZ patients (Csernansky et al. 1998, 2002; Wang et al. 2001; Shenton et al. 2002; Zierhut et al. 2013; Mamah et al. 2016). However, brain differences detected by MRI are influenced by multiple factors, some with potentially opposing effects, including the duration of exposure to antipsychotic medication (Lieberman et al. 2005; Ho et al. 2011). Dissecting the effects of disease and other moderating factors, such as medication exposure in clinical MRI studies is therefore extremely difficult.

Data from the ENIGMA Schizophrenia Working Group ( $n=2028$ SCZ patients and $n=2540$ controls) suggests that decreases in hippocampal volume are positively associated with the proportion of unmedicated SCZ patients (van Erp et al. 2015). This suggests that

This is an Open Access article, distributed under the terms of the Creative Commons Attribution licence (http://creativecommons.org/licenses/by/4.0/), which permits unrestricted re-use, distribution, and reproduction in any medium, provided the original work is properly cited. 
structural abnormalities of the hippocampus may be more severe in untreated patients and potentially, those volume deficits may be ameliorated, at least partially, by treatment with antipsychotic drugs (APD) (Szeszko et al. 2003; Narr et al. 2004; van Erp et al. 2015). In the recent ENIGMA dataset, 11 of the study sample sites included patients predominantly treated with second-generation (atypical) antipsychotics (SGA) (van Erp et al. 2015). Importantly, longitudinal MRI studies, which have the advantage of controlling for baseline changes prior to medication exposure, report significant decreases from baseline in either the grey-matter volume, or the shape of the temporal lobe and the hippocampus in SCZ patients treated with first-generation antipsychotics (FGA), but not SGA (Lieberman et al. 2005; McClure et al. 2006, 2008, 2013; Koolschijn et al. 2010). A more recent study replicated the finding of reduced temporal lobe grey-matter volume, but this effect was independent of APD treatment (Ho et al. 2011). In addition, higher doses of quetiapine are associated with decreased hippocampal grey-matter volume (Ebdrup et al. 2011). In parallel to volume studies, longitudinal analysis of shape deformations in the hippocampus reveals that these are present in ultra-high-risk individuals and persist following transition to psychosis (Dean et al. 2016). Furthermore, inward shape deformation of the posterior hippocampus appears to predict the degree of positive symptoms in these patients (Dean et al. 2016). Longitudinal studies have also attempted to examine the potential influence of antipsychotics on hippocampal shape. Mamah et al. (2012) report progressive hippocampal shape abnormalities between SCZ patients treated with haloperidol (HAL), compared to olanzapine (OLZ) (Mamah et al. 2012). Shape deformation of the CA1 region in SCZ patients is also reported to predict the dose of APD required to treat positive symptoms (Zierhut et al. 2013). Conversely, at least four longitudinal studies report no significant relationships between antipsychotic use and hippocampal volume or shape (Arango et al. 2003; Velakoulis et al. 2006; Panenka et al. 2007; McClure et al. 2013). However, the lack of longitudinally followed untreated patients as a control means it remains unclear whether these outcomes are the effect of illness progression, antipsychotic treatment or an interaction between the two. Further, none of the human studies have linked the imaging changes to post-mortem findings and therefore the relationship between imaging-related structural changes and postmortem findings remains unclear (Vernon et al. 2011). Thus, whilst exposure to APD have been linked to both hippocampal volume and shape changes in SCZ patients, the findings are clearly equivocal.

Pre-clinical studies incorporating rodent MRI have previously been useful to address this issue, providing evidence that chronic exposure to either HAL or OLZ leads to alterations in brain volume (Vernon et al. 2011, 2012, 2014). To date, however, there has been no investigation of the effect of chronic APD exposure on shape metrics, although this is possible using rodent MR images (Delgado y Palacios et al. 2011; Wheeler et al. 2013). In the current study, we set out to address this question by utilizing ex vivo MRI data available from our prior study of the effects of chronic exposure to APD on rat brain volume (Vernon et al. 2011). Specifically, we tested for the presence of hippocampal volume differences per voxel, using tensorbased morphometry (TBM) in male rats exposed for 8 weeks to either HAL ( $2 \mathrm{mg} / \mathrm{kg}$ per day) or OLZ (10 $\mathrm{mg} / \mathrm{kg}$ per day), compared to vehicle (VEH)-treated controls. TBM is a sensitive and observer-independent measure of brain atrophy, which is well suited to rodent MRI studies where the grey- to white- matter ratio is low (Lau et al. 2008; Lerch et al. 2008; Vernon et al. 2014; Harrison et al. 2015). In parallel, we applied a novel elastic surface-based shape analysis method to search for deformations in hippocampal shape as a function of chronic APD exposure. Based on our prior observations that chronic exposure to either HAL or OLZ did not lead to a decrease in hippocampal volume, using manual segmentation analysis, we hypothesized that the more sensitive TBM analysis may potentially disprove this finding. In terms of shape metrics, since the available clinical data are equivocal, we made no specific a priori hypothesis.

\section{Materials and method}

\section{Animals}

This study used ex vivo MR images and brain tissue sections collected from VEH- and APD-exposed animals, as previously reported (Vernon et al. 2011) (see also Supplementary materials and methods). No new animals were generated for this study. Briefly, a common VEH ( $\beta$-hydroxypropylcyclodextrin, $20 \% \mathrm{w} / \mathrm{v}$, acidified by ascorbic acid to $\mathrm{pH} 6 ; n=8), \mathrm{HAL}(2 \mathrm{mg} / \mathrm{kg}$ per day; $n=8$; Sigma-Aldrich, UK), or OLZ (10 mg/kg per day; $n=8$; Biophore Pharmaceuticals Ltd, India) were administered to experimentally naive, 10-week-old male Sprague-Dawley rats (Charles River, UK), using subcutaneously implanted osmotic minipumps for a total of 8 weeks (Vernon et al. 2011). Osmotic minipumps (Alzet Model 2ML4, 28 days; Alzet, USA) filled with drug or VEH solutions were inserted subcutaneously on the back flank under isoflurane anaesthesia (5\% induction, $1.5 \%$ maintenance) and replaced once after 28 days. Animals were habituated for 7 days before experimental procedures, which were carried out in accordance with the Home Office Animals (Scientific procedures) Act, 
United Kingdom, 1986 and European Union Directive 2010/63/EU.

\section{MRI acquisition}

Brains were prepared for ex vivo MR imaging as previously described (Vernon et al. 2011, 2014) (see also Supplementary materials and methods). Briefly, MR image acquisition was performed using a $7 \mathrm{~T}$ horizontal small bore magnet (Varian, USA) with custom-made quadrature volume radiofrequency $(\mathrm{RF})$ coil $(43 \mathrm{~mm}$ inner diameter, Rapid Biomedical GmbH, Germany) connected to a console running VnmrJ acquisition software (v. 2.3; Varian). A modified multiecho, multislice spin-echo pulse sequence was used for image acquisition, with the following parameters: field of view $=35 \times 35$ $\mathrm{mm}^{2}$; matrix $=192 \times 192$; repetition time $=4200 \mathrm{~ms}$; echo time $=10,20,30,40,50,60,70,80 \mathrm{~ms} ; 8$ averages, 50 slices, $0.5 \mathrm{~mm}$ thick, with a total duration of $2 \mathrm{~h} 30 \mathrm{~min}$ and an in plane resolution of $187 \mu \mathrm{m}$.

\section{Assessment of hippocampal volume using TBM}

A mean image of the entire dataset ( $n=24$ scans) was generated using rigid-body registration ( 6 d.f.) using a population-based registration method based on FSL-FLIRT (Jenkinson \& Smith, 2001; Jenkinson et al. 2002; Crum et al. 2013). Using this mean image, the external and internal borders of the left and right hippocampus (dorsal and ventral regions, approximately to -1.92 to $-6.84 \mathrm{~mm}$ from bregma based on the rat stereotaxic atlas (Watson \& Paxinos, 2007) were manually defined using the polygon tool in ITK-SNAP (http://www.itksnap.org) (Yushkevich et al. 2006) by an expert rater (A.C.V.) using previously published criteria (Wolf et al. 2002; Vernon et al. 2011) (Fig.1a, b). Segmentation performance was assessed using intra-class correlation coefficient with values $<0.95$ rejected. This segmentation was used to create a binary mask for implementation in the TBM pipeline (Fig. 1c). Tensor-based morphometry was used to assess anatomical differences related to APD treatment in the hippocampus as previously described (Vernon et al. 2014) (see also Supplementary materials and methods). Using high-dimensional non-rigid fluid registration, the binary hippocampus mask defined in the template space was warped back onto individual MR images using the inverse of the transformation and used for subsequent shape analysis.

\section{Assessment of hippocampal shape using elastic surface-based shape deformation analysis}

The shape analysis pipeline has been reported previously (Danckaers et al. 2014) and is depicted in Fig. 2a. Changes in the shape and size of the hippocampus may be described by computing the inward and outward displacement vectors from the surface of the structure (Delgado y Palacios et al. 2011; Wheeler et al. 2013). First, a reference hippocampal surface is registered to a target hippocampal surface, by minimizing the geometric distance between those surfaces while maintaining correspondences. Here, the binary hippocampus mask (reference) is warped to match the population average image of all rats (target). Fig. $2 b$ shows the geometric error (Euclidean distance) between the original surface and the registered surface, calculated for every surface and the average error projected on to the average surface. From this final nonlinear atlas, a surface representation of the hippocampus is generated. Due to the anisotropy resulting from the $2 \mathrm{D}$ acquisition (in-slice $v$. axial resolution), the resulting 3D hippocampal surfaces exhibit a stairlike structure. Therefore, in order to obtain a more accurate 3D surface while still interpolating the actual slices, the surfaces were prepared by interpolating the distance transform of adjacent slices over a regular grid. The global rigid registration and an elasticitymodulated registration are then iteratively repeated ( $n=60$ iterations). During the iterations, the stiffness gradually decreases, such that the surface will become more elastic through the iterations. The first step of surface registration is the application of a rigid alignment. To that end, in both hippocampal surfaces corresponding points are identified, by casting a normal ray from each vertex of the reference surface to the target surface. When the normal of an intersection point is in the same direction, within a tolerance of $60^{\circ}$, as the normal of the point on the reference surface, that point can be considered corresponding. This tolerance is based on the dot product between the normal of the source vertex and the intersection of this normal with the target surface. If this dot product $>0.5$, those points are considered as corresponding points. Another restriction for corresponding points is that the normal may not intersect the surface multiple times before reaching the corresponding point. Once corresponding point sets are obtained, they are used to rigidly align the surfaces in a least squares sense using singular value decomposition.

In the elastic part of the registration, the vertices are allowed to translate separately, while motion is restricted by a stiffness parameter, which regulates the strength of the connection with the neighbouring vertices and which decreases during the iterations. The stiffness parameter is $\beta$ in the pipeline (Fig. 2a) and regulates how stiff or elastic the surface can behave. When this parameter is high, the neighbouring vertices of a translated vertex are forced to move (partially) along. In contrast, when this parameter is low, the surface is more elastic and the connection between 
(a)

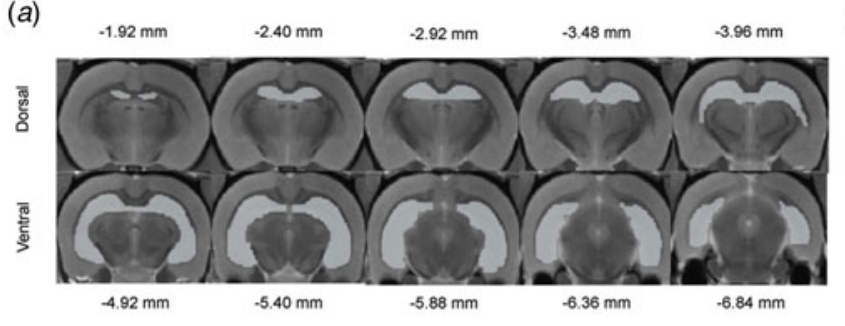

(b)

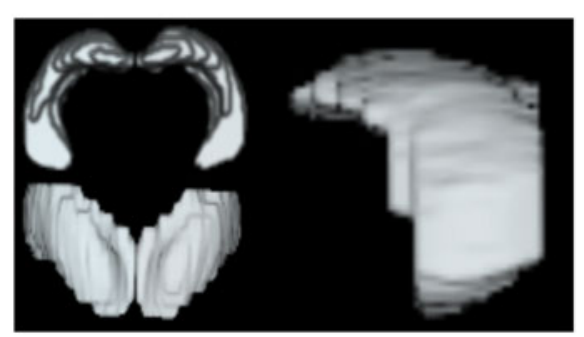

(c)

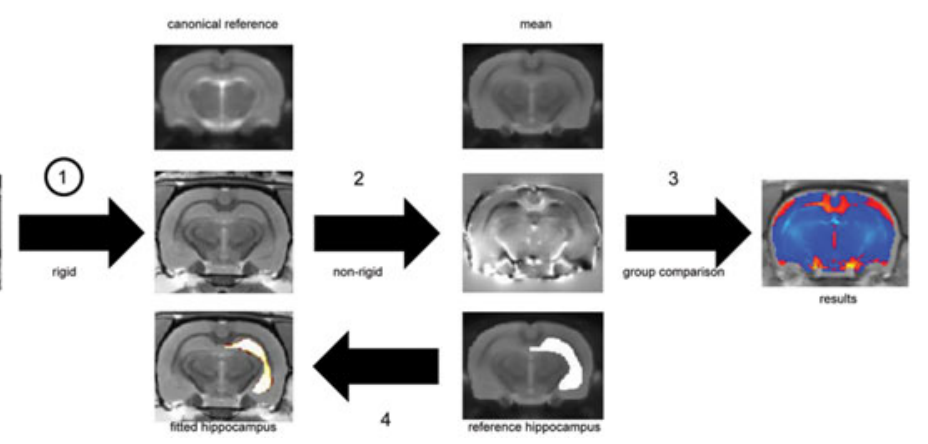

Fig. 1. (a) Anatomical criteria for creation of the binary mask for tensor-based morphometry (TBM) and shape analysis of the rat hippocampus using a mean MR image of the entire dataset ( $n=24$ scans). The orientation of the displayed scans is in the coronal plane. (b) Three-dimensional rendering of the binary mask of the rat hippocampus. (c) Processing pipeline for automated TBM analysis.

(a)
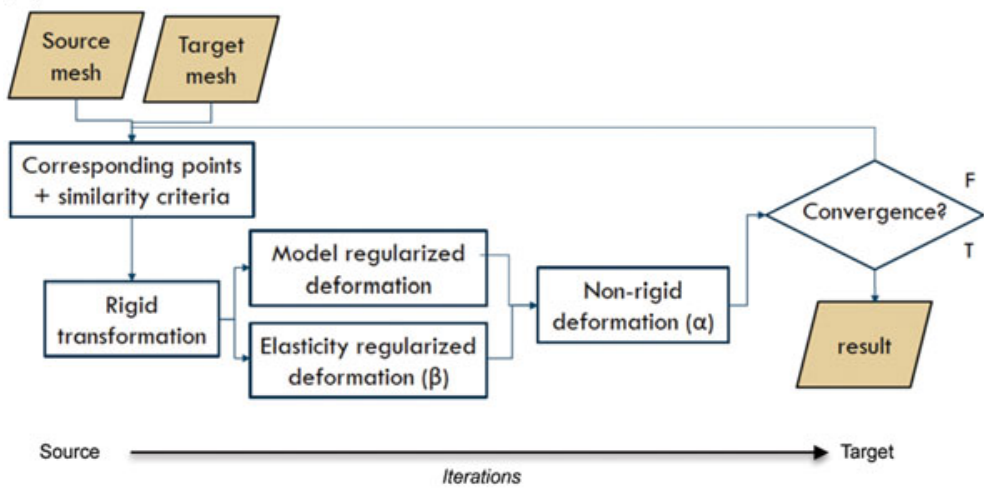

(b)

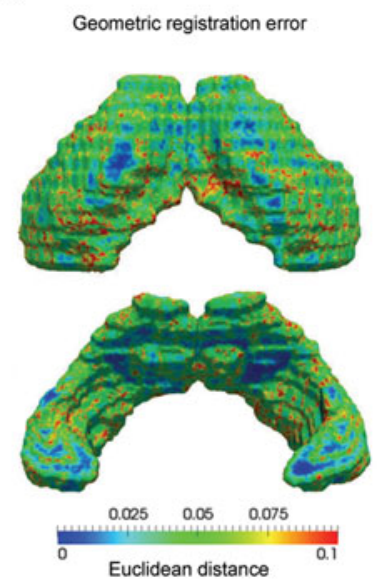

Fig. 2. (a). Image processing pipeline for elastic surface-based shape deformation analysis. (b) The geometric error (Euclidean distance) between the original surface and the registered surface. This error is calculated for every surface and the average error is projected on the average surface and displayed with a colour map.

neighbouring vertices is lower, such that these may move more freely. By applying weights to each vertex, the importance of this vertex can be set. If no corresponding point for a vertex of the source mesh can be found, its weight is set to zero. In that case, this vertex simply translates along with its neighbouring vertices. When all surfaces are registered by the same reference surface, each vertex has the same anatomical location on each hippocampal surface.

\section{Post-mortem tissue handling and Cavalieri probe analysis of hippocampal volume}

After MRI acquisition, brains were removed from the skull and processed for Nissl staining as previously described (Vernon et al. 2011) (see also Supplementary materials and methods). A single observer (M.C.C.) blinded to experimental group by coding measured the volume of the hippocampus using the Cavalieri 
estimator (Gundersen \& Jensen, 1987) as described elsewhere (Vernon et al. 2011) (see also Supplementary materials and methods).

\section{Statistical analysis}

Voxel-wise analyses of local volume changes for group differences were computed using a one-way ANOVA followed by voxel-wise $t$ tests to compare VEH to each antipsychotic drug (VEH v. HAL; VEH v. OLZ), as well as each antipsychotic to the other (HAL $v$. OLZ) (Vernon et al. 2014). Shape changes were analysed per vertex by comparing the vector lengths and direction, performing a covariance calculation using non-parametric permutation tests based on Hoteling's T2 statistics. For both volume and shape analysis, multiple comparisons were controlled for using the FDR at $q=0.05$ (Genovese et al. 2002). Due to the small sample size in the current study, the data were also analysed at an exploratory threshold of $p<0.05$ uncorrected for multiple comparisons. Post-mortem volume data were analysed using one-way ANOVA with Bonferroni post-hoc test using SPSS v. 22.0 software (IBM Corp., USA).

\section{Ethical standards}

The authors assert that all procedures contributing to this work comply with the ethical standards of the relevant national and institutional guides on the care and use of laboratory animals.

\section{Results}

Chronic APD exposure has no significant effect on hippocampal volume in naive rats

The TBM analysis revealed no significant effects (FDR, $q=0.05$ ) of chronic exposure (8 weeks) to either HAL or OLZ on hippocampal volume, in either the left or right brain hemisphere (Supplementary Fig. S1A, B). This replicates our previous null findings using manual segmentation (Vernon et al. 2011). As the sample size was small, which may compromise our ability to detect subtle changes, we carried out an additional exploratory analysis $(p<0.05$ uncorrected; (Vernon et al. 2014). However, even at this exploratory threshold we could not detect any clusters of contracted or expanded voxels in our hippocampal region of interest (data not shown).

\section{Impact of chronic APD exposure on hippocampal shape deformation}

The results of the shape analysis are shown in Fig. $3 a-c$. It is clear from the data that there are both net outward and inward vertex displacements in hippocampal shape following chronic exposure to either antipsychotic compared to VEH-treated animals. Chronic HAL exposure resulted in significant $(q<0.05$, FDR-corrected; Fig. 3a) inward displacement in the dorsal hippocampus compared to VEH-treated rats. These displacements are bilateral, but appear more pronounced in the left hemisphere of the brain. These inward displacements correspond approximately to the cornu ammonis 1 (CA) and sub-field, extending through the oriens, pyramidal and radiatum layers to touch the CA3 sub-field. Additional significant, bilateral inward deformations were also found in the CA3 sub-field in the ventral hippocampus. In contrast, significant outward deformations were observed in both hemispheres of the ventral hippocampus. These are again bilateral, but predominate in the right hemisphere and correspond approximately to the CA1 and CA2 sub-fields.

Chronic OLZ exposure also led to significant $(q<$ 0.05; FDR-corrected; Fig. $3 b$ ) deformations in the dorsal and ventral hippocampus compared to VEH-exposed rats. Although these deformations overlap topographically with those observed in HAL-exposed animals, specifically to the left dorsal CA1 sub-field and right ventral CA1 and CA2 sub-fields, they are less widespread (Fig. 3a, b). Furthermore, the dorsal CA1 deformations in OLZ-exposed animals are in the opposite direction to those in HAL-exposed animals, moving outward, rather than inward, relative to $\mathrm{VEH}$ controls (Fig. 3a,b). When directly comparing shape differences between HAL- and OLZ-exposed animals, this effect shows up as a subtle, but significant difference between the two APD (Fig. 3c). Interestingly however, in the ventral aspect of the hippocampus in OLZ-exposed rats, there is vertex displacement that overlaps both in terms of topography and direction of change with those observed in HAL-exposed animals, although these did not survive multiple comparisons correction (Fig. 3b).

The lack of TBM changes strongly suggests the volume of the hippocampus is not altered across treatment groups and that these deformations represent true shape changes. However, this can also be tested directly using volume normalization in order to visualize the pure shape differences. In Fig. 4, the volume of the hippocampal surfaces is normalized such that each hippocampus has a size of 1 . When comparing the data before and after volume normalization, the results are identical, consistent with the lack of volume change detected by TBM or manual segmentation. These data strongly suggest that we are detecting true shape changes in the hippocampus following chronic exposure to APD. The presence of both inward and outward shape deformations is therefore also consistent with the failure to find significant differences in 
(a)
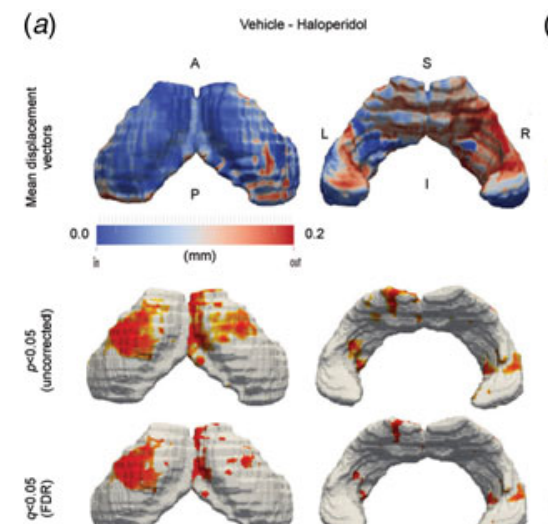

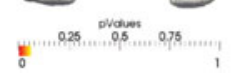

(b)
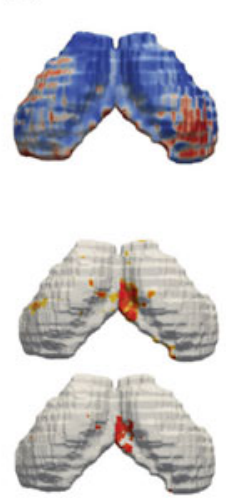

Vehicle - Olanzapine
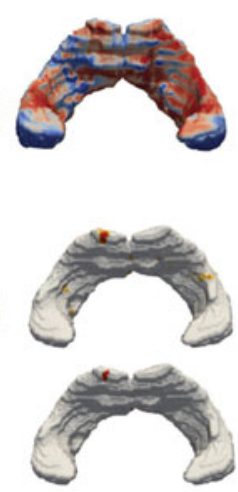

$(c)$
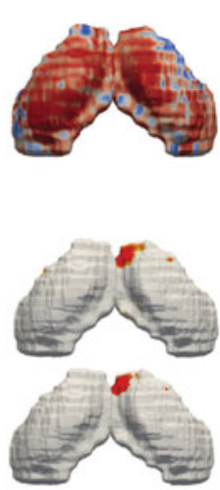

operidol - Olanzapine

Fig. 3. Elastic shape deformation analysis reveals common and divergent effects of chronic haloperidol and olanzapine exposure on hippocampal shape metrics. Data shown are the mean inward and outward displacement vectors ( $\mathrm{mm}$ ), shown both at an exploratory threshold of $p<0.05$ uncorrected for multiple comparisons and $q=0.05$ FDR-corrected, to illustrate significant differences in hippocampal shape metrics between $(a)$ vehicle and haloperidol; $(b)$ vehicle and olanzapine and $(c)$ haloperidol and olanzapine exposed rats. Directionality of statistically significant hippocampal shape deformations, either inward (blue) or outward (red), may be found by comparing the $p$ value maps with the mean displacement vector maps in each treatment group comparison. A, Anterior; P, posterior; S, superior; I, inferior; L, left; R, right.

(a)
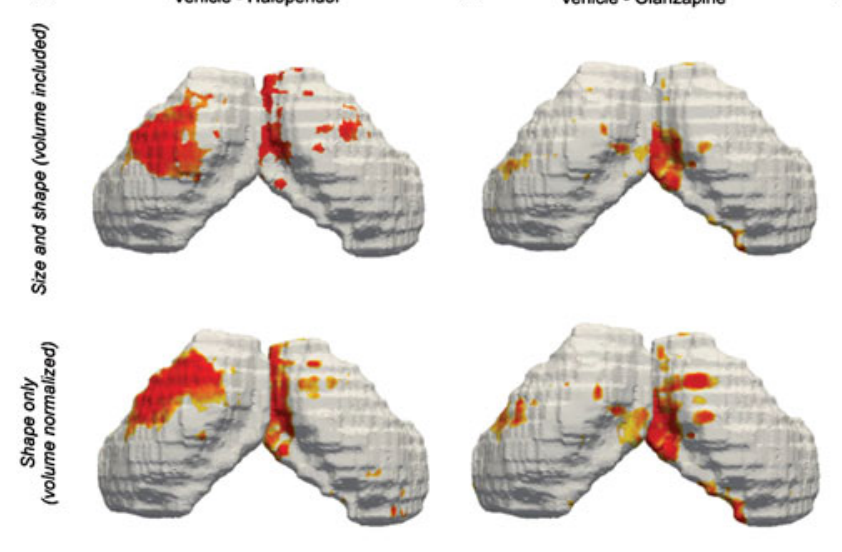

(b)

Vehicle - Olanzapine

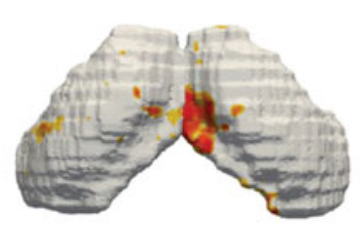

(c)

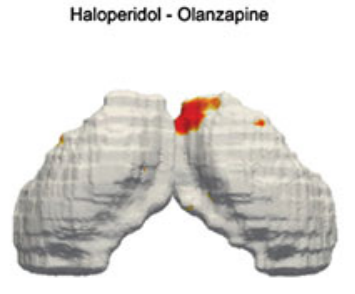

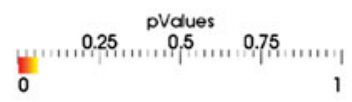

Fig. 4. Normalization of hippocampal volume does not alter the pattern of significant voxels identified by elastic shape deformation analysis, suggesting true shape changes. Data shown are size and shape and shape only (volume normalized to 1 ) for $(a)$ vehicle $v$. haloperidol; $(b)$ vehicle $v$. olanzapine and $(c)$ haloperidol $v$. olanzapine.

volume, either by TBM or manual segmentation, since the net hippocampal volume change may be very close to zero.

\section{Confirmation of the absence of hippocampal volume changes post-mortem}

Post-mortem volume analysis using the Cavalieri probe revealed no significant overall differences in the total volume (dorsal + ventral) of the left hippocampus between treatment groups $\left(F_{2,21}=0.1 .75, p>0.05\right.$; Fig. 5 and Supplementary Table S1).

\section{Discussion}

Contrary to our hypothesis, TBM analysis confirmed that chronic exposure (8 weeks) to either HAL or 


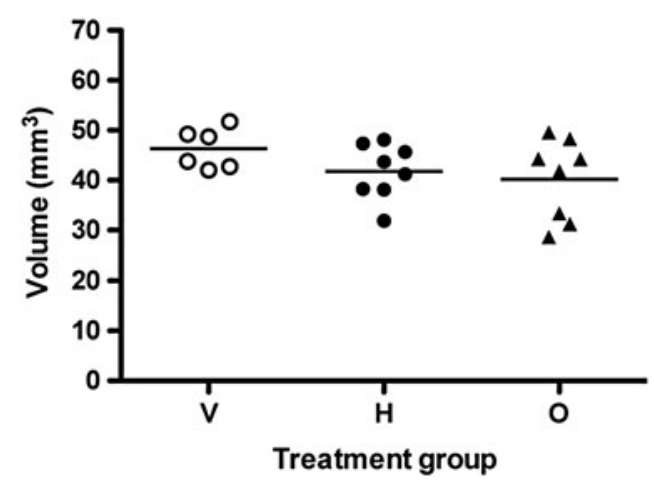

Fig. 5. Post-mortem confirmation of the lack of hippocampal volume changes in rats chronically exposed to either haloperidol or olanzapine using the Cavalieri probe. Data shown are individual hippocampal volume (scatter plot with mean) in vehicle $(\mathrm{V})$, haloperidol $(\mathrm{H})$ and olanzapine $(\mathrm{O})$ treated rats.

OLZ, at clinically relevant doses, does not lead to changes in the volume of the rat hippocampus. In contrast, the results of the shape analysis reveal that chronic exposure to either HAL or OLZ results in common shape changes both in terms of their topography and direction, particularly in the ventral hippocampus. However, the data also provide preliminary evidence for minor divergent shape changes following exposure to these two different antipsychotics, particularly in the CA1 sub-field of the rat dorsal hippocampus. In this region, while the drug-induced shape changes overlap topographically, HAL exposure leads to inward deformations, whilst OLZ leads to outward deformations. Furthermore, hippocampal shape changes appear more widespread following HAL treatment, particularly again in the dorsal hippocampus. These data raise new questions about our interpretation of clinical MRI data and how antipsychotics might impact hippocampal structure in SCZ patients.

In the largest cross-sectional MR imaging dataset available comparing healthy controls and SCZ patients, including both medicated and un-medicated individuals (ENIGMA; van Erp et al. 2015), the volume of the hippocampus in SCZ patients was significantly reduced (Cohen's $d=-0.46$ ). This reduction was more pronounced in patients who were not medicated (van Erp et al. 2015). Interestingly, the majority of medicated patients were treated with SGA. Although the FGA/ SGA nomenclature is a poorly defined and heterogeneous construct (Leucht et al. 2009), prior studies suggest there may be distinct effects of different APD on brain volume. For example cortical volume (Lieberman et al. 2005; Ansell et al. 2015) and cortical thickness (van Haren et al. 2011) are reported to be less apparent in SCZ patients treated with SGA, including OLZ. A recent meta-analysis of longitudinal structural MRI studies in medicated SCZ patients also suggests SGA have less impact on brain volume, compared to FGA, including HAL (Vita et al. 2015). In contrast, primate (Dorph-Petersen et al. 2005) and rodent (Vernon et al. 2011) pre-clinical studies have failed to find significant differences between the effects of HAL and OLZ on brain volume following chronic exposure, including no effect on hippocampal volume, using manual segmentation (Vernon et al. 2011). The results of the current study using operatorindependent voxel-wise TBM analysis, which has greater sensitivity for subtle anatomical changes confirm our prior in vivo data. Furthermore, we corroborate the TBM analysis post-mortem using unbiased stereology analysis of hippocampal volume from Nissl stained tissue sections. The lack of hippocampal volume change following APD exposure may reflect the fact that these are naive animals, which do not replicate pathological conditions relevant to SCZ. Alternatively, since the hippocampal shape deformations are both inward and outward, the net volume change may be close to zero. These data are, however, consistent with the majority of hippocampal volume MRI studies in patients with SCZ which find no relationship between antipsychotic dose and hippocampal volume (Marsh et al. 1994; Whitworth et al. 1998; Stefanis et al. 1999; Altshuler et al. 2000; Gur et al. 2000; Arango et al. 2003; Szeszko et al. 2003; Narr et al. 2004; Lieberman et al. 2005; Velakoulis et al. 2006; Panenka et al. 2007; Koolschijn et al. 2010; Ho et al. 2011).

Importantly, shape analysis complements volumetric analysis and can identify subtle regional abnormalities in brain structures in the absence of overall volumetric changes (Csernansky et al. 1998; Csernansky et al. 2002). The current study therefore provides the first preclinical evidence that chronic exposure to HAL or OLZ leads to common, but also distinct effects on hippocampal shape. Specifically, chronic treatment with both drugs led to topographically overlapping shape deformations, which were clearly more extensive in HAL than OLZ treated animals. Interestingly, in the dorsal CA1 sub-field, HAL exposure led to inward deformation, while OLZ promoted outward deformation. In contrast, both HAL and OLZ had similar effects in the ventral hippocampus, promoting predominantly outward deformations, although it is notable these did not survive FDR correction in OLZ-exposed rats. These data are partly consistent with findings from a longitudinal MRI study of first-episode SCZ patients randomized to either HAL or OLZ, which suggested that OLZ treatment was associated with a significantly lower percentage of 'large magnitude negative surface vertex slopes' as compared to HAL treatment, when patients were followed up to 104 weeks (Mamah et al. 
2012). Notably however, other studies on the same dataset found no effect of antipsychotic exposure at any follow-up time point (McClure et al. 2006, 2008, 2013). This may be explained by the differential shape analysis methods employed in these studies, which could influence the results (Mamah et al. 2016). Our preclinical data suggest the dorsal hippocampus is a potential locus of differential shape changes, whilst the ventral hippocampus shows common shape changes following chronic treatment with either HAL or OLZ in the naive rat brain. Although preliminary, these data suggest a testable hypothesis for future studies with a larger sample size to confirm these data. This could also provide a dataset for the controlled testing of the effects of different shape analysis methodology, which may also be beneficial to the field.

It is conceivable however, that the observed hippocampal shape changes may reflect volume changes in adjacent brain regions, which cause an alteration in hippocampal shape rather than volume. In our prior work, we have shown that chronic antipsychotic exposure decreases total cortical volume, but primarily in the cingulate and somatosensory cortex (Vernon et al. 2011, 2014). Chronic HAL exposure also leads to striatal enlargement (Vernon et al. 2012). However, as yet we do not have information regarding the effects of chronic antipsychotic exposure on the shape or volume of other sub-cortical nuclei in close proximity to the hippocampus, in particular, the thalamus. Further studies using both TBM and shape analysis are required to assess the potential influence of alterations in volume or shape metrics in these structures on the present findings in the hippocampus.

What might be the cellular origin of these APDinduced hippocampal shape changes? Rigorous postmortem studies in macaque monkeys using clinically comparable plasma levels, chronic antipsychotic treatment, and unbiased stereology reported a significant $20 \%$ decrease in the total number of $S 100 \beta+$ astrocytes albeit in the grey matter of the left parietal lobe, but no change in neuronal number (Konopaske et al. 2008). These provide immediately testable hypothesis for future studies in the rat brain. Using post-mortem brain tissue from the same animals from which the current imaging data were generated, we have recently reported that chronic HAL and OLZ exposure resulted in highly significant increases in the density of amoeboid Iba1+ microglia in both the dorsal and ventral rat hippocampus (Cotel et al. 2015). These effects on microglia thus overlap topographically with the areas of shape change in the rat hippocampus after chronic HAL or OLZ exposure. Furthermore, both HAL and OLZ have been previously reported to impact on growth factors, particularly nerve growth factor (NGF), brain-derived neurotrophic factor (BDNF) and fibroblast growth factor (FGF) (Pillai \& Mahadik, 2006; Pillai et al. 2006). Moreover, oxidative stress in the hippocampus has been reported following HAL, but not OLZ treatment (Reinke et al. 2004). Altogether, changes in these parameters could lead to alterations in axon sprouting, fibre reorganization, myelin formation; neurogenesis, dendritic spine morphology (Uranova et al. 1991) and angiogenesis, all of which reflect potential contributors to the observed hippocampal shape changes.

The clinical impact of these APD-induced shape changes also remains unclear. Indeed, it is challenging to label the directionality of our findings as 'beneficial' or 'harmful' (Lewis, 2011). The human (or primate) hippocampus may be functionally divided along its anterioposterior axis into an anterior part, which is involved in emotional processing and a posterior part, which is predominantly involved in memory function. In contrast, the rat hippocampus is orientated along a dorsal-ventral axis, divided into the dorsal and ventral sections, which are also functionally dissociated (Moser \& Moser, 1998; Fanselow \& Dong, 2010). Specifically, the dorsal part of the rat hippocampus contributes to cognitive functions, including spatial learning and memory and is therefore analogous to the human (or primate) posterior hippocampus (Bannerman et al. 2004; McHugh et al. 2004). In contrast, the rat ventral hippocampus is involved in the regulation of stress, anxiety and emotional memory and is therefore analogous to the human (or primate) anterior hippocampus (Barkus et al. 2010). As such, both divisions of the hippocampus show differential connectivity patterns. For example, memory function is critically dependent upon connections between the dorsal hippocampus and retrosplenial granular and anterior cingulate cortices (ACC) (Fanselow \& Dong, 2010). Patients with SCZ exhibit non-specific hyperactivity of the left anterior hippocampus during a longterm memory task, which is related to positive symptom severity (Zierhut et al. 2010). Furthermore, ultrahigh risk and first-episode psychosis individuals demonstrate increased cerebral blood flow specifically in the anterior CA1 sub-field of the hippocampus, which correlates with atrophy of this region and predicts transition to psychosis (Schobel et al. 2009; Schobel et al. 2013). Several studies have also identified inward shape deformations in the anterior and posterior aspects of the human hippocampus, which are correlated with the extent of positive symptoms in SCZ patients (Csernansky et al. 2002; Small et al. 2011; Zierhut et al. 2013; Dean et al. 2016; Mamah et al. 2016). Inward shape deformations of the anterior and posterior CA1 sub-field also predicts daily antipsychotic drug dosage in SCZ patients (Zierhut et al. 2013; Dean et al. 2016). Shape deformations of the CA1 sub-fi 
eld, may therefore be the consequence of a diseasespecific hyperactive hippocampal state, leading to elevated glutamate levels, downstream atrophy and disruption of CA1 functional connectivity, resulting in the symptomatology of SCZ (Schobel et al. 2009; Schobel et al. 2013; Zierhut et al. 2013). Speculatively, the common outward shape deformations we observe in the ventral hippocampus, the rat homologue of the human anterior hippocampus following chronic exposure to either HAL or OLZ, may reflect a structural consequence of alterations in hippocampus/CA1 activity or functional connectivity, which may be related to the therapeutic effects of these medications on positive symptoms, through correction of aberrant brain activity patterns. In other words, a beneficial effect related to the therapeutic action of the drug.

In contrast, these APD-induced hippocampal shape deformations may be related to adverse motor and cognitive side-effects of APD. Notably, we previously observed common effects of both HAL and OLZ to decrease ACC volume, which were more apparent in HAL-treated rats (Vernon et al. 2014). These data may suggest a link between hippocampal shape deformations, ACC volume and functional connectivity of these structures following chronic antipsychotic drug treatment, which requires exploration. Although recent studies have begun to examine the complex network effects of antipsychotics on brain functional connectivity (Gass et al. 2013; Wheeler et al. 2014), no study has to date linked these to morphometric changes or behavioural outcomes following chronic dosing. Of possible relevance to this study however, Gass et al. (2013) reported decreased functional connectivity between the substantia nigra and the CA2 region of the dorsal hippocampus in rats following acute HAL challenge (Gass et al. 2013). Further studies are required combining functional and structural MR imaging to address whether alterations in brain activity are related to structural remodelling following chronic APD exposure. Importantly, there is a paucity of preclinical studies examining the relationship between chronic APD exposure using clinically relevant dosing and the cognitive or emotional functions ascribed to the dorsal and ventral aspects of the rodent hippocampus. Although prior studies have not typically used mini-pump delivery methods, acute and chronic treatment with various APD leads to impairment in Morris water-maze tasks of spatial learning but not other maze tasks, such as the elevated plus maze or radial arm maze (Skarsfeldt, 1996; Didriksen et al. 2006; Terry \& Mahadik, 2007; Hutchings et al. 2013). Further studies are therefore required to understand the links between dorsal and ventral hippocampus shape deformations, cognitive and emotional behaviour, particularly any differences, or indeed, similarities, following exposure to HAL or OLZ.
It should be noted, however, that we examined male rats only. This is relevant, since a prior study reported a significant decrease in hippocampal volume, following chronic OLZ exposure in female SD rats (Barr et al. 2013). Interestingly, this decrease was related to metabolic side effects of chronic OLZ exposure, in this case, impaired glucose tolerance and increased peripheral adiposity (Barr et al. 2013). Notably, we have previously reported that the OLZ-exposed animals used in this study have lower hepatic levels of Insulin receptor substrate 2 (IRS2), indicative of impaired insulin signalling and gluco-metabolic abnormalities (Mondelli et al. 2013). However, these animals did not show weight gain or increased adiposity as compared to VEH controls (Mondelli et al. 2013). In contrast, the HALexposed rats showed increase adiposity, in the absence of weight gain and no change in hepatic IRS2 protein levels (Mondelli et al. 2013). These data suggest that different molecular pathways mediate the disturbances of glucose homeostasis induced by HAL and OLZ, at least in male rats; nonetheless, metabolic abnormalities were clearly present in both antipsychotic exposed groups. However, female rats produce more consistent and perhaps larger, metabolic changes than males in response to antipsychotic exposure (Boyda et al. 2010) which may contribute to reduced hippocampal volume (Barr et al. 2013). However, the study by Barr and colleagues also used different dosing methods (daily injection $v$. osmotic minipumps in the current study), which may affect the data by virtue of differential pharmacokinetics (Kapur et al. 2003). Nevertheless, investigations into potential sex differences in the effects of APD on brain structure and function, particularly in relation to peripheral metabolic side effects of these drugs are clearly required.

Importantly, the current experiments were conducted in healthy rats, free from pathology, in order to define the effects of chronic antipsychotic exposure on shape and volume metrics in a controlled fashion. Ultimately, however, to begin to establish whether these drug-induced hippocampal shape changes are either beneficial or harmful, will require repetition of these experiments in rat models that model relevant aspects of SCZ pathology. Specifically, these could include MR-detectable increases in hippocampal activity and associated decreases in the volume of the hippocampus such as those observed following either prenatal exposure to maternal infection (Piontkewitz et al. 2011) or chronic N-methyl-D-aspartate receptor antagonism (Schobel et al. 2013; Barnes et al. 2015). This may well reveal important differences, including any drug $x$ disease interactions and bring us closer to a clearer vision of the potential clinical implications of these findings. Further caveats regarding the interpretation of our findings should be also be 
acknowledged. The use of hippocampal surface models relies on manual tracing, and systematic bias in delineating this region might adversely affect its reliability. However, the reliability of the segmentation method has been shown to be high (Wolf et al. 2002). Although the tracings were performed blinded to treatment group, any differences in image contrast between $\mathrm{VEH}$ and antipsychotic-treated rats could also potentially affect the results. Nevertheless, we have not detected significant changes in T2 signal intensity in other brain regions that displayed volume changes after chronic APD treatment (Vernon et al. 2011). The resolution of our MR images is relatively low and the original images were not isotropic. It is therefore possible that partial volume effects or imperfections in image registration may be driving the observed effects of HAL and OLZ on hippocampal shape. Although the registration error between the source and target images was low (see Fig. 2b), it will be important to confirm our observations by collecting higher resolution ex vivo MR images with isotropic voxels from $\mathrm{VEH}$ and antipsychotic-treated rats. The sample size is small ( $n=8$ per group) and our findings thus require confirmation in a larger sample size. Notably, we have however replicated the effects of HAL on cerebral morphometry observed in these same animals (Vernon et al. 2011) in a larger sample size of $n=12$ per group (Vernon et al. 2012). We tested only one dose of each antipsychotic drug, which although within the range of striatal $\mathrm{D}_{2}$ receptor occupancy similar to levels obtained in clinical settings (Kapur et al. 2003) a full dose response study may be beneficial.

In conclusion, we show for the first time that chronic treatment of naive adult rats with either HAL or OLZ lead to changes in hippocampal shape metrics, in the absence of volume changes. Strikingly, the effects of both drugs were relatively common, particularly in the ventral hippocampus. Nevertheless, our data also suggest preliminary evidence for differential shape changes following HAL or OLZ exposure in the dorsal hippocampus, which require confirmation in a larger sample size. The effects were more profound with HAL, as compared to OLZ, consistent with recent clinical observations. It is however, important to stress that these data were collected in normal rats, which does not reflect the innate pathology of SCZ. Furthermore, because the cellular basis and functional consequences of these effects remain unknown, one should be cautious in drawing clinical inferences. Nonetheless, our studies provide a clear rationale for future experiments to explicitly examine the relationships between changes in hippocampal shape metrics and how these relate to the efficacy of APD as well as morphological, cognitive and metabolic side effects of chronic APD treatment.

\section{Supplementary material}

For supplementary material accompanying this paper visit http://dx.doi.org/10.1017/S0033291716001768.

\section{Acknowledgements}

This work was supported by the Medical research Council (grant numbers G0701748, G1002198). The authors acknowledge the British Heart Foundation for supporting the $7 \mathrm{~T}$ MRI scanner at the King's College London Preclinical imaging unit (KCLPIU).

\section{Declaration of Interest}

Doctors Danckaers, Crum, Huysmans, Cotel, Natesan, Modo, Vernon and Professor Sijbers and Professor Williams have no conflicting interests to declare. Professor Kapur has received grant support from AstraZeneca and GSK, and has served as consultant and/or speaker for AstraZeneca, Bioline, BMS-Otsuka, Eli Lilly, Janssen (J\&J), Lundbeck, NeuroSearch, Pfizer, Roche, Servier and Solvay Wyeth in the last 3 years.

\section{References}

Altshuler LL, Bartzokis G, Grieder T, Curran J, Jimenez T, Leight K, Wilkins J, Gerner R, Mintz J (2000). An MRI study of temporal lobe structures in men with bipolar disorder or schizophrenia. Biological Psychiatry 48, 147-162.

Ansell BR, Dwyer DB, Wood SJ, Bora E, Brewer WJ, Proffitt TM, Velakoulis D, McGorry PD, Pantelis C (2015). Divergent effects of first-generation and second-generation antipsychotics on cortical thickness in first-episode psychosis. Psychological Medicine 45, 515-527.

Arango C, Breier A, McMahon R, Carpenter Jr. WT, Buchanan RW (2003). The relationship of clozapine and haloperidol treatment response to prefrontal, hippocampal, and caudate brain volumes. American Journal of Psychiatry 160, 1421-1427.

Bannerman DM, Rawlins JN, McHugh SB, Deacon RM, Yee BK, Bast T, Zhang WN, Pothuizen HH, Feldon J (2004). Regional dissociations within the hippocampus - memory and anxiety. Neuroscience Biobehavioural Reviews 28, 273-283.

Barkus C, McHugh SB, Sprengel R, Seeburg PH, Rawlins JN, Bannerman DM (2010). Hippocampal NMDA receptors and anxiety: at the interface between cognition and emotion. European Journal of Pharmacology 626, 49-56.

Barnes SA, Sawiak SJ, Caprioli D, Jupp B, Buonincontri G, Mar AC, Harte MK, Fletcher PC, Robbins TW, Neill JC, Dalley JW (2015). Impaired limbic cortico-striatal structure and sustained visual attention in a rodent model of schizophrenia. International Journal of Neuropsychopharmacology 18, 1-12.

Barr AM, Wu CH, Wong C, Hercher C, Topfer E, Boyda HN, Procyshyn RM, Honer WG, Beasley CL (2013). Effects of chronic exercise and treatment with the antipsychotic drug 
olanzapine on hippocampal volume in adult female rats. Neuroscience 255, 147-157.

Boyda HN, Tse L, Procyshyn RM, Honer WG, Barr AM (2010). Preclinical models of antipsychotic drug-induced metabolic side effects. Trends in Pharmacological Sciences 31, 484-497.

Cotel MC, Lenartowicz EM, Natesan S, Modo MM, Cooper JD, Williams SC, Kapur S, Vernon AC (2015). Microglial activation in the rat brain following chronic antipsychotic treatment at clinically relevant doses. European

Neuropsychopharmacology 25, 2098-2107.

Crum WR, Modo M, Vernon AC, Barker GJ, Williams SC (2013). Registration of challenging pre-clinical brain images. Journal of Neuroscience Methods 216, 62-77.

Csernansky JG, Joshi S, Wang L, Haller JW, Gado M, Miller JP, Grenander U, Miller MI (1998). Hippocampal morphometry in schizophrenia by high dimensional brain mapping. Proceedings of the National Academy Science USA 95, 11406-11411.

Csernansky JG, Wang L, Jones D, Rastogi-Cruz D, Posener JA, Heydebrand G, Miller JP, Miller MI (2002).

Hippocampal deformities in schizophrenia characterized by high dimensional brain mapping. American Journal of Psychiatry 159, 2000-2006.

Danckaers F, Huysmans T, Lacko D, Ledda A, Verwulgen S, Van Dongen S, Sijbers J (2014). Correspondance preserving elastic surface registration with shape model prior. Pattern Recognition (International Conference on Pattern Recognition) 22, 2143-2148.

Dean DJ, Orr JM, Bernard JA, Gupta T, Pelletier-Baldelli A, Carol EE, Mittal VA (2016). Hippocampal shape abnormalities predict symptom progression in neuroleptic-free youth at ultrahigh risk for psychosis. Schizophrenia Bulletin 42, 161-169.

Delgado y Palacios R, Campo A, Henningsen K, Verhoye M, Poot D, Dijkstra J, Van Audekerke J, Benveniste H, Sijbers J, Wiborg O, Van der Linden A (2011). Magnetic resonance imaging and spectroscopy reveal differential hippocampal changes in anhedonic and resilient subtypes of the chronic mild stress rat model. Biological Psychiatry 70, 449-457.

Didriksen M, Kreilgaard M, Arnt J (2006). Sertindole, in contrast to clozapine and olanzapine, does not disrupt water maze performance after acute or chronic treatment. European Journal Pharmacology 542, 108-115.

Dorph-Petersen KA, Pierri JN, Perel JM, Sun Z, Sampson AR, Lewis DA (2005). The influence of chronic exposure to antipsychotic medications on brain size before and after tissue fixation: a comparison of haloperidol and olanzapine in macaque monkeys. Neuropsychopharmacology 30, 1649-1661.

Ebdrup BH, Skimminge A, Rasmussen H, Aggernaes B, Oranje B, Lublin H, Baare W, Glenthoj B (2011). Progressive striatal and hippocampal volume loss in initially antipsychotic-naive, first-episode schizophrenia patients treated with quetiapine: relationship to dose and symptoms. International Journal of Neuropsychopharmacology 14, 69-82.

Fanselow MS, Dong HW (2010). Are the dorsal and ventral hippocampus functionally distinct structures? Neuron 65, 7-19.
Gass N, Schwarz AJ, Sartorius A, Cleppien D, Zheng L, Schenker E, Risterucci C, Meyer-Lindenberg A, Weber-Fahr W (2013). Haloperidol modulates midbrain-prefrontal functional connectivity in the rat brain. European Neuropsychopharmacology 23, 1310-1319.

Genovese CR, Lazar NA, Nichols T (2002). Thresholding of statistical maps in functional neuroimaging using the false discovery rate. Neuroimage 15, 870-878.

Gundersen HJ, Jensen EB (1987). The efficiency of systematic sampling in stereology and its prediction. Journal of Microscopy 147, 229-263.

Gur RE, Turetsky BI, Cowell PE, Finkelman C, Maany V, Grossman RI, Arnold SE, Bilker WB, Gur RC (2000). Temporolimbic volume reductions in schizophrenia. Archives of General Psychiatry 57, 769-775.

Harrison IF, Crum WR, Vernon AC, Dexter DT (2015). Neurorestoration induced by the HDAC inhibitor sodium valproate in the lactacystin model of Parkinson's is associated with histone acetylation and up-regulation of neurotrophic factors. British Journal of Pharmacology 172, 4200-4215.

Ho BC, Andreasen NC, Ziebell S, Pierson R, Magnotta V (2011). Long-term antipsychotic treatment and brain volumes: a longitudinal study of first-episode schizophrenia. Archives of General Psychiatry 68, 128-137.

Hutchings EJ, Waller JL, Terry Jr AV (2013). Differential long-term effects of haloperidol and risperidone on the acquisition and performance of tasks of spatial working and short-term memory and sustained attention in rats. Journal of Pharmacology and Experimental Therapeutics 347, 547-556.

Jenkinson M, Bannister P, Brady M, Smith S (2002). Improved optimization for the robust and accurate linear registration and motion correction of brain images. Neuroimage 17, 825-841.

Jenkinson M, Smith S (2001). A global optimisation method for robust affine registration of brain images. Medical Image Analysis 5, 143-156.

Kapur S, VanderSpek SC, Brownlee BA, Nobrega JN (2003). Antipsychotic dosing in preclinical models is often unrepresentative of the clinical condition: a suggested solution based on in vivo occupancy. Journal of Pharmacology Experimental Therapeutics 305, 625-631.

Konopaske GT, Dorph-Petersen KA, Sweet RA, Pierri JN, Zhang W, Sampson AR, Lewis DA (2008). Effect of chronic antipsychotic exposure on astrocyte and oligodendrocyte numbers in macaque monkeys. Biological Psychiatry 63, 759-765.

Koolschijn PC, van Haren NE, Cahn W, Schnack HG, Janssen J, Klumpers F, Hulshoff Pol HE, Kahn RS (2010). Hippocampal volume change in schizophrenia. Journal of Clinical Psychiatry 71, 737-744.

Lau JC, Lerch JP, Sled JG, Henkelman RM, Evans AC, Bedell BJ (2008). Longitudinal neuroanatomical changes determined by deformation-based morphometry in a mouse model of Alzheimer's disease. Neuroimage 42, 19-27.

Lerch JP, Carroll JB, Spring S, Bertram LN, Schwab C, Hayden MR, Henkelman RM (2008). Automated 
deformation analysis in the YAC128 Huntington disease mouse model. Neuroimage 39, 32-39.

Leucht S, Corves C, Arbter D, Engel RR, Li C, Davis JM (2009). Second-generation versus first-generation antipsychotic drugs for schizophrenia: a meta-analysis. Lancet 373, 31-41.

Lewis DA (2011). Antipsychotic medications and brain volume: do we have cause for concern? Archives of General Psychiatry 68, 126-127.

Lieberman JA, Tollefson GD, Charles C, Zipursky R, Sharma T, Kahn RS, Keefe RS, Green AI, Gur RE, McEvoy J, Perkins D, Hamer RM, Gu H, Tohen M (2005). Antipsychotic drug effects on brain morphology in first-episode psychosis. Archives of General Psychiatry 62, 361-370.

Mamah D, Alpert KI, Barch DM, Csernansky JG, Wang L (2016). Subcortical neuromorphometry in schizophrenia spectrum and bipolar disorders. Neuroimage Clinical 11, 276-286.

Mamah D, Harms MP, Barch D, Styner M, Lieberman JA, Wang L (2012). Hippocampal shape and volume changes with antipsychotics in early stage psychotic illness. Frontiers in Psychiatry 3, 96.

Marsh L, Suddath RL, Higgins N, Weinberger DR (1994). Medial temporal lobe structures in schizophrenia: relationship of size to duration of illness. Schizophrenia Research 11, 225-238.

McClure RK, Carew K, Greeter S, Maushauer E, Steen G, Weinberger DR (2008). Absence of regional brain volume change in schizophrenia associated with short-term atypical antipsychotic treatment. Schizophrenia Research 98, 29-39.

McClure RK, Phillips I, Jazayerli R, Barnett A, Coppola R, Weinberger DR (2006). Regional change in brain morphometry in schizophrenia associated with antipsychotic treatment. Psychiatry Research 148, 121-132.

McClure RK, Styner M, Maltbie E, Lieberman JA, Gouttard S, Gerig G, Shi X, Zhu H (2013). Localized differences in caudate and hippocampal shape are associated with schizophrenia but not antipsychotic type. Psychiatry Research 211, 1-10.

McHugh SB, Deacon RM, Rawlins JN, Bannerman DM (2004). Amygdala and ventral hippocampus contribute differentially to mechanisms of fear and anxiety. Behavioural Neuroscience 118, 63-78.

Mondelli V, Anacker C, Vernon AC, Cattaneo A, Natesan S, Modo M, Dazzan P, Kapur S, Pariante CM (2013). Haloperidol and olanzapine mediate metabolic abnormalities through different molecular pathways. Translational Psychiatry 3, e208.

Moser MB, Moser EI (1998). Functional differentiation in the hippocampus. Hippocampus 8, 608-619.

Narr KL, Thompson PM, Szeszko P, Robinson D, Jang S, Woods RP, Kim S, Hayashi KM, Asunction D, Toga AW, Bilder RM (2004). Regional specificity of hippocampal volume reductions in first-episode schizophrenia. Neuroimage 21, 1563-1575.

Panenka WJ, Khorram B, Barr AM, Smith GN, Lang DJ, Kopala LC, Vandorpe RA, Honer WG (2007). A longitudinal study on the effects of typical versus atypical antipsychotic drugs on hippocampal volume in schizophrenia. Schizophrenia Research 94, 288-292.

Pillai A, Mahadik SP (2006). Differential effects of haloperidol and olanzapine on levels of vascular endothelial growth factor and angiogenesis in rat hippocampus. Schizophrenia Research 87, 48-59.

Pillai A, Terry Jr AV, Mahadik SP (2006). Differential effects of long-term treatment with typical and atypical antipsychotics on NGF and BDNF levels in rat striatum and hippocampus. Schizophrenia Research 82, 95-106.

Piontkewitz Y, Arad M, Weiner I (2011). Abnormal trajectories of neurodevelopment and behavior following in utero insult in the rat. Biological Psychiatry 70, 842-851.

Reinke A, Martins MR, Lima MS, Moreira JC, Dal-Pizzol F, Quevedo J (2004). Haloperidol and clozapine, but not olanzapine, induces oxidative stress in rat brain. Neuroscience Letters 372, 157-160.

Schobel SA, Chaudhury NH, Khan UA, Paniagua B, Styner MA, Asllani I, Inbar BP, Corcoran CM, Lieberman JA, Moore H, Small SA (2013). Imaging patients with psychosis and a mouse model establishes a spreading pattern of hippocampal dysfunction and implicates glutamate as a driver. Neuron 78, 81-93.

Schobel SA, Lewandowski NM, Corcoran CM, Moore H, Brown T, Malaspina D, Small SA (2009). Differential targeting of the CA1 subfield of the hippocampal formation by schizophrenia and related psychotic disorders. Archives of General Psychiatry 66, 938-946.

Shenton ME, Gerig G, McCarley RW, Szekely G, Kikinis R (2002). Amygdala-hippocampal shape differences in schizophrenia: the application of 3D shape models to volumetric MR data. Psychiatry Research 115, 15-35.

Skarsfeldt T (1996). Differential effect of antipsychotics on place navigation of rats in the Morris water maze. A comparative study between novel and reference antipsychotics. Psychopharmacology (Berlin) 124, 126-133.

Small SA, Schobel SA, Buxton RB, Witter MP, Barnes CA (2011). A pathophysiological framework of hippocampal dysfunction in ageing and disease. Nature Reviews Neuroscience 12, 585-601.

Stefanis N, Frangou S, Yakeley J, Sharma T, O'Connell P, Morgan K, Sigmudsson T, Taylor M, Murray R (1999). Hippocampal volume reduction in schizophrenia: effects of genetic risk and pregnancy and birth complications. Biological Psychiatry 46, 697-702.

Szeszko PR, Goldberg E, Gunduz-Bruce H, Ashtari M, Robinson D, Malhotra AK, Lencz T, Bates J, Crandall DT, Kane JM, Bilder RM (2003). Smaller anterior hippocampal formation volume in antipsychotic-naive patients with first-episode schizophrenia. American Journal of Psychiatry 160, 2190-2197.

Terry Jr AV, Mahadik SP (2007). Time-dependent cognitive deficits associated with first and second generation antipsychotics: cholinergic dysregulation as a potential mechanism. Journal of Pharmacology and Experimental Therapeutics 320, 961-968.

Uranova NA, Orlovskaya DD, Apel K, Klintsova AJ, Haselhorst U, Schenk H (1991). Morphometric study of synaptic patterns in the rat caudate nucleus and 
hippocampus under haloperidol treatment. Synapse 7, 253-259.

van Erp TG, Hibar DP, Rasmussen JM, Glahn DC, Pearlson GD, Andreassen OA, Agartz I, Westlye LT, Haukvik UK, Dale AM, Melle I, Hartberg CB, Gruber O, Kraemer B, Zilles D, Donohoe G, Kelly S, McDonald C, Morris DW, Cannon DM, Corvin A, Machielsen MW, Koenders L, de Haan L, Veltman DJ, Satterthwaite TD, Wolf DH, Gur RC, Gur RE, Potkin SG, Mathalon DH, Mueller BA, Preda A, Macciardi F, Ehrlich S, Walton E, Hass J, Calhoun VD, Bockholt HJ, Sponheim SR, Shoemaker JM, van Haren NE, Pol HE, Ophoff RA, Kahn RS, Roiz-Santianez R, Crespo-Facorro B, Wang L, Alpert KI, Jonsson EG, Dimitrova R, Bois C, Whalley HC, McIntosh AM, Lawrie SM, Hashimoto R, Thompson PM, Turner JA (2015). Subcortical brain volume abnormalities in 2028 individuals with schizophrenia and 2540 healthy controls via the ENIGMA consortium. Molecular Psychiatry 21, 547-553.

van Haren NE, Schnack HG, Cahn W, van den Heuvel MP, Lepage C, Collins L, Evans AC, Hulshoff Pol HE, Kahn RS (2011). Changes in cortical thickness during the course of illness in schizophrenia. Archives of General Psychiatry 68, 871-880.

Velakoulis D, Wood SJ, Wong MT, McGorry PD, Yung A, Phillips L, Smith D, Brewer W, Proffitt T, Desmond P, Pantelis C (2006). Hippocampal and amygdala volumes according to psychosis stage and diagnosis: a magnetic resonance imaging study of chronic schizophrenia, first-episode psychosis, and ultra-high-risk individuals. Archives of General Psychiatry 63, 139-149.

Vernon AC, Crum WR, Lerch JP, Chege W, Natesan S, Modo M, Cooper JD, Williams SC, Kapur S (2014). Reduced cortical volume and elevated astrocyte density in rats chronically treated with antipsychotic drugs-linking magnetic resonance imaging findings to cellular pathology. Biological Psychiatry 75, 982-990.

Vernon AC, Natesan S, Crum WR, Cooper JD, Modo M, Williams SC, Kapur S (2012). Contrasting effects of haloperidol and lithium on rodent brain structure: a magnetic resonance imaging study with postmortem confirmation. Biological Psychiatry 71, 855-863.

Vernon AC, Natesan S, Modo M, Kapur S (2011). Effect of chronic antipsychotic treatment on brain structure: a serial magnetic resonance imaging study with ex vivo and postmortem confirmation. Biological Psychiatry 69, 936-944.

Vita A, De Peri L, Deste G, Barlati S, Sacchetti E (2015). The effect of antipsychotic treatment on cortical gray matter changes in Schizophrenia: does the class matter? A meta-analysis and meta-regression of longitudinal magnetic resonance imaging studies. Biological Psychiatry 78, 403-412.

Wang L, Joshi SC, Miller MI, Csernansky JG (2001). Statistical analysis of hippocampal asymmetry in schizophrenia. Neuroimage 14, 531-545.

Watson C, Paxinos G (2007). The Rat Brain in Stereotaxic Co-Ordinates. Academic Press: London.

Wheeler AL, Creed MC, Voineskos AN, Nobrega JN (2014). Changes in brain functional connectivity after chronic haloperidol in rats: a network analysis. International Journal of Neuropsychopharmacology 17, 1129-1138.

Wheeler AL, Lerch JP, Chakravarty MM, Friedel M, Sled JG, Fletcher PJ, Josselyn SA, Frankland PW (2013). Adolescent cocaine exposure causes enduring macroscale changes in mouse brain structure. Journal of Neuroscience 33, 1797-1803.

Whitworth AB, Honeder M, Kremser C, Kemmler G, Felber S, Hausmann A, Wanko C, Wechdorn H, Aichner F, Stuppaeck CH, Fleischhacker WW (1998). Hippocampal volume reduction in male schizophrenic patients. Schizophrenia Research 31, 73-81.

Wolf OT, Dyakin V, Vadasz C, de Leon MJ, McEwen BS, Bulloch K (2002). Volumetric measurement of the hippocampus, the anterior cingulate cortex, and the retrosplenial granular cortex of the rat using structural MRI. Brain Research Brain Research Protocols 10, 41-46.

Yushkevich PA, Piven J, Hazlett HC, Smith RG, Ho S, Gee JC, Gerig G (2006). User-guided 3D active contour segmentation of anatomical structures: significantly improved efficiency and reliability. Neuroimage 31, 1116-1128.

Zierhut K, Bogerts B, Schott B, Fenker D, Walter M, Albrecht D, Steiner J, Schutze H, Northoff G, Duzel E, Schiltz K (2010). The role of hippocampus dysfunction in deficient memory encoding and positive symptoms in schizophrenia. Psychiatry Research 183, 187-194.

Zierhut KC, Grassmann R, Kaufmann J, Steiner J, Bogerts B, Schiltz K (2013). Hippocampal CA1 deformity is related to symptom severity and antipsychotic dosage in schizophrenia. Brain 136, 804-814. 